THE JOURNAL OF

THOBAGIG

GARDIOVASEULAR

SUREERY

Vol. 135, No. 3, March 2008

\title{
Envisioning simulation in the future of thoracic surgical education
}

Andrea J. Carpenter, MD, PhD, ${ }^{\text {a }}$ Stephen C. Yang, MD, ${ }^{b}$ Paul N. Uhlig, MD, ${ }^{c}$ and Yolonda L. Colson, MD, PhD

From the Division of Thoracic Surgery, University of Texas Health Science Center at San Antonio, San Antonio, Tex ${ }^{\mathrm{a}}$; the Division of Thoracic Surgery, Johns Hopkins Medical Institutions, Baltimore, $\mathrm{Md}^{\mathrm{b}}$; Central Plains Cardiothoracic Surgery LLC, Wichita, Kans ${ }^{\mathrm{c}}$; and the Division of Thoracic Surgery, Department of Surgery, Brigham and Women's Hospital, Boston, Mass. ${ }^{\text {d }}$

Writing for the participants of the Visioning Simulation Conference, convened at the Center for Medical Simulation, Cambridge, Mass, April 19-20, 2007.

Received for publication Dec 18, 2007; accepted for publication Dec 18, 2007.

Address for reprints: Andrea J. Carpenter, MD, PhD, Division of Thoracic Surgery, 7703 Floyd Curl Dr MSC-7841, San Antonio, TX 78229-3900 (E-mail: carpenterA2@ uthscsa.edu).

J Thorac Cardiovasc Surg 2008;135:477-84 $0022-5223 / \$ 34.00$

Copyright $\odot 2008$ by The American Association for Thoracic Surgery

doi:10.1016/j.jtcvs.2007.12.005
7 he Visioning Simulation Conference (VSC), convened in Cambridge, Mass, on April 19, 2007, sponsored by the Thoracic Surgery Foundation for Research and Education (TSFRE), was attended by appointed representatives from the Society of Thoracic Surgery (STS), the American Association for Thoracic Surgery (AATS), the American Board of Thoracic Surgery (ABTS), the Thoracic Surgery Directors Association (TSDA), the European Association for Cardiothoracic Surgery (EACTS), the American College of Surgeons (ACS), the National Heart, Lung, and Blood Institute (NHLBI), industry partners (Medtronic, Inc, Edwards Lifesciences LLC, and St Jude Medical, Inc), and several attendees with extensive simulation experience and development. The conference began with a simulated operating room (OR) demonstration by live video feed. During the 2-day conference, speakers discussed simulation use in anesthesia, the airline industry, and congenital heart disease, with the goal of demonstrating what technology is currently available and stimulating thought among the participants regarding future needs. The primary working function of the conference centered on a series of roundtable discussions planned to define how simulation could be applied to cardiothoracic surgical education. This report defines the vision developed during this 2-day conference and recommends actions necessary to bring this vision to reality.

Surgical education is becoming a progressively more complex endeavor. In this era of limited work hours for residents and rapidly changing technology, new and creative educational techniques must be used to ensure that surgeons in training achieve proficiency in more complex problems during shorter training periods and that practicing surgeons can be rapidly trained in new technologies. It is clear that simulation will become a required method for demonstrating proficiency, maintaining certification, and introducing new techniques. Evolving requirements for measurable quality indicators will soon mandate that maintenance of certification require the demonstration of proficiency in technical skills, fundamental knowledge, and management of complex clinical situations.

The VSC was based on the premise that simulation could be used as a tool to meet these needs in thoracic surgery. Under the auspices of TSFRE and the Joint Council on Thoracic Surgical Education (JCTSE), a committee was formed to plan the VSC. The objective of the conference was to bring together surgeons, professionals with simulation experience, simulation developers, government, and industry funding partners. The committee devised a program that combined simulation demonstrations, lectures by simulation experts, and a series of roundtable discussions. The primary focus of the conference centered on the following questions:

- How can simulation be used to improve patient safety and transform thoracic surgery in a positive way?

- What is unique about thoracic surgery as a specialty that makes it appropriate for simulation education? 
- Considering current and emerging technologies, which procedures and patient care activities most need to be simulated?

- Which team challenges and competencies most need to be simulated in the OR environment and in the critical care environment?

- What key simulation activities should happen, and in which locations?

- Whose support and what resources are necessary to begin implementation of simulation in thoracic surgery, and how do we access them?

Appointed representatives from STS, AATS, ABTS, TSDA, EACTS, ACS, NHLBI, and industry partners (Medtronic, Inc, Edwards Lifesciences LLC, and St Jude Medical, Inc) attended the VSC. In addition, several attendees with extensive simulation experience and development were active participants, including members of the airline industry, simulation developers, and engineers. The primary goal of the VSC was to develop a vision of simulation as an educational tool for thoracic surgery and to outline a strategy for moving forward with implementation.

\section{How can simulation be used to improve patient safety and transform thoracic surgery in a positive way?}

Historically, surgical training has followed an apprenticeship model. The resident begins operating with a senior surgeon and learns by doing the operation and gradually assuming more responsibility for each portion of the procedure. Industries that have evolved simulation as a valuable training tool have moved away from this model. No passenger would ride on a commercial flight during a pilot's first experience in the cockpit; thus, completion of simulator training is required for every commercial pilot. In the same manner, simulation can transform the process of learning thoracic surgery while posing far less risk to our patients.

Individual skills at all levels are an obvious target for simulation. Very basic skills, such as constructing an anastomosis, placing cannulas, or identifying and exposing intrathoracic structures, could be simulated with currently available low-fidelity technology. Simulation could also evolve to identify an aptitude, or lack thereof, for cardiothoracic surgery. Exposure to simulated scenarios could help potential candidates make an informed decision about important career choices. Simultaneously, this experience could help to identify the best candidates for subsequent training.

Pattern recognition is also an integral part of every thoracic operation and is particularly important when a procedure requires multitasking. For example, simulation of the physiologic events associated with instituting, monitoring, and separating from cardiopulmonary bypass (CPB) can teach a trainee to recognize appropriate patterns of response. Once these patterns have been learned, various alterations can be introduced into the simulations to help the trainee recognize an event that does not follow the usual pattern, identify the aberration, and learn corrective measures.

Team simulation can expose all members of the surgical team to the entire process of patient care. Simulated scenarios would permit the teaching of surgeons at all levels, including critical nontechnical skills such as the fine art of communicating with patients and families, practicing the delivery of "bad news," understanding personal biases, and assessing individual communication skills. Communication failure is the most common cause of litigation; thus, the value of improving this skill cannot be overestimated. Team training effectively improves communication among team members and helps individuals recognize their roles within the team. Scenarios with emphasis on quality indicators can improve the efficiency of perioperative procedures, present rare events to the team before they are encountered in the OR, and model activities surrounding patient transport and handoff to train the team to reduce or eliminate errors during this crucial exchange.

Simulation is also an ideal way to evaluate new technologies or novel surgical approaches, identifying potential shortcomings before use in the OR with a live patient. Threedimensional computer graphic modeling can be used to define patient anatomy and provide insight into the anatomic variations that may be encountered. A data repository that could be accessed by surgeons worldwide would provide views of infrequently encountered anatomic structures, which would be especially helpful in training surgeons specializing in congenital heart disease. Magnetic resonance imaging reconstructions can clearly recreate individual anatomy and permit patient-specific planning of surgical procedures. Once a new technique is well developed, simulation of the new procedure could permit improved dissemination of the technology and "cross-fertilization" of ideas between centers. Fully trained surgeons could use simulator training to learn new procedures before performing them on patients. Prime examples of such skills are endovascular techniques with associated imaging.

Lessons learned from the aviation industry have proved the value of simulation for certification. Maintaining certification with regular experience in the simulated environment could truly transform our profession. VSC participants repeatedly discussed and emphasized, however, the importance of providing excellent fidelity and validation of simulations before implementation to determine certification status.

\section{What is unique about cardiothoracic surgery as a specialty that makes it appropriate for simulation education?}

Cardiothoracic surgeons have a tendency to assume leadership, as evident in their choice of a highly complex and demanding profession. In addition, the practice of cardiothoracic surgery involves extensive use of technology, much of which is ideally suited to simulation. Simulating CPB with monitor feedback of physiologic responses, providing tactile 
feedback from a location remote from the surgeon's hands in robotic surgery, and mimicking endovascular skills and associated imaging are all situations in which simulation can provide the surgeon with valuable experience.

The reality of the VSC clearly demonstrates the commitment of the cardiothoracic surgical leadership to this endeavor. Representatives from multiple societies, organizations, and specialty interests came together for 2 days to concentrate on the use of simulation to advance thoracic surgical education and confirmed the intent to move the field forward through the use of simulation. The conference provided an opportunity to learn from the experiences of other industries, to use existing technology, and to imagine new ways of using simulation to our advantage.

\section{Considering current and emerging technologies, which procedures and patient care activities most need to be simulated?}

The list of individual procedures performed by cardiothoracic surgeons is extensive. Some skills are universal whereas, others are more aligned with the specific subspecialties of adult cardiac surgery, congenital cardiac surgery, and general thoracic surgery (Table 1). The specific needs for simulation unique to each subspecialty are discussed here.

\section{Adult Cardiac Surgery}

Scenarios involving initiation of and separation from CPB can be used to teach individual residents or to train teams. Such simulations can include challenges that will teach problem solving or evaluate an individual surgeon's problemsolving skills. Examples of such challenges are cannulation site dissection, management of the porcelain aorta or mobile aortic atheroma, left ventricular dilation during weaning, wall motion abnormalities, and acute electrocardiographic changes. Simulation of uncommon surgical misadventures could provide experience that would greatly enhance patient safety. Good examples are reentry challenges, such as anterior pseudoaneurysm, management of graft injury, and reconstruction of atrioventricular groove dissociation.

Similarly, infrequently encountered procedures should also be simulated, thereby increasing the experience of rare cases for all surgeons. The simulator would be helpful in teaching techniques for intracardiac repairs, such as mitral valve exposure and mitral valve reconstruction, repair of postinfarct ventricular septal defect, or left ventricular reconstruction for heart failure. Simulation could be used to teach the best methods of performing the maze atrial fibrillation procedures and to simulate ventricular assist device implantation and decannulation techniques.

New techniques can be rapidly and safely incorporated into adult cardiac surgery through the use of simulation. Alternative surgical access techniques are increasing in popularity and use; such techniques include partial valve replacement or repairs and robotic procedures through a sternotomy or minithoracotomy. These techniques could be perfected in a simulated environment before being performed for the first time on a patient. A trainer for off-pump coronary bypass surgery would be invaluable in teaching maneuvers that can help stabilize the heart and the vital signs while exposing all areas of the heart. Use of the simulator to teach trainees how to construct an anastomosis with difficult exposure or imperfect stabilization would clearly improve patient outcomes. Currently available endovascular simulators can be used to teach fundamental endovascular catheter skills. These simulators can help surgeons learn to observe and evaluate 2dimensional fluoroscopic images as 3-dimensional events. Endovascular simulators can be very useful both in teaching endovalve procedures and transapical valve procedures and in developing future valve replacement and repair techniques.

\section{Congenital Cardiac Surgery}

Surgery for congenital heart disease presents the surgeon with a unique challenge. There are so many variations of each congenital anomaly that the individual surgeon will see only a fraction of the possibilities in a lifetime, making the learning of procedures and development of new techniques very difficult. Beginning with magnetic resonance images of the heart, 3-dimensional imaging techniques show individual variations in anatomy and allow manipulation that very closely simulates operative findings. This technology should be pursued with two goals: (1) building a repository of images that can be viewed and manipulated by surgeons in all surgical centers dealing with congenital heart disease and (2) constructing patient-specific images that can aid in operative planning. Once an anatomic repository has been built, physiologic flow dynamic display could be added.

By building computer imaging simulation and imaging databases, surgeons will be better able to develop newer operative techniques for correction. As new procedures evolve and are validated, the techniques can more readily be disseminated to centers worldwide. Ultimately, such dissemination can improve patient outcomes and increase safety by eliminating, or at least reducing, the trial and error involved in perfecting these procedures on patients with complicated anatomy.

\section{General Thoracic Surgery}

Interventional endoscopy is viewed as a vulnerable area that may be lost to other specialties. It is therefore important both to provide training in these techniques during residency and to introduce these techniques to practicing surgeons. Simulation can be a valuable tool for helping surgeons learn unfamiliar techniques, including laser application, stent placement, and ultrasound interpretation.

Simulation is also a viable option for disseminating newer techniques to thoracic surgeons. Pulmonary resections by video-assisted thoracic surgery (VATS) and laparoscopic approaches to esophageal procedures are clear examples. Looking to the future, as VATS and laparoscopy become 
Table 1. Short- and long-term simulation goals in each of the three subspecialties

\begin{tabular}{|c|c|c|}
\hline Subspecialty & Short term (12-18 mo) & Long term (3-5 y) \\
\hline Adult cardiac & $\begin{array}{l}\text { First Tier } \\
\text { CPB cannulation } \\
\text { Circulatory arrest technique } \\
\text { Off-pump CABG } \\
\text { Hybrid procedures } \\
\text { CABG (on and off CPB) } \\
\text { Endovascular grafts, wires, and complications } \\
\text { Second Tier } \\
\text { Catheter-based valves } \\
\text { ICU modules } \\
\text { Neuroprotection method } \\
\text { Cardiac wire skills (pacemaker, ICD) } \\
\text { LVAD implantation (ventricular support) } \\
\text { Robotic procedures (MV, ITA) } \\
\text { Valve repair }\end{array}$ & $\begin{array}{l}\text { Uncommon intracardiac procedures (eg, septal myectomy, maze) } \\
\text { Intraop management of heart failure } \\
\text { Coronary procedures (stents, angiography) } \\
\text { Team training (emergency situations, preop planning, basic setup) } \\
\text { Intraop problems: reentry, cannulation, groove destruction } \\
\text { Robotics: MV repair, ITA preparation }\end{array}$ \\
\hline General Thoracic & $\begin{array}{l}\text { Procedures } \\
\text { Interventional bronchoscopy or EGD } \\
\text { (laser, EBUS) } \\
\text { Stent and catheter placement } \\
\text { VATS (lobectomy, thymectomy) } \\
\text { Laparoscopy (fundoplication) } \\
\text { Chest tube } \\
\text { Intubating difficult airway } \\
\text { Mediastinoscopy } \\
\text { Patient Care } \\
\text { Communication skills (OR, nursing, ICU) } \\
\text { Team leading } \\
\text { Preop Issues } \\
\text { Communication preparation } \\
\text { Informed consent } \\
\text { Practice management } \\
\text { Risk assessment } \\
\text { Preop preparation } \\
\text { Managing cases } \\
\text { Intraop Issues } \\
\text { Judgment scenarios } \\
\text { Bleeding } \\
\text { Complications } \\
\text { Vascular control } \\
\text { Postop Issues } \\
\text { Debriefing } \\
\text { Discussing death } \\
\text { Advanced disease } \\
\text { Withdrawing care } \\
\text { Complication management (fistula, } \\
\text { arrhythmias, pain, persistent air leak) }\end{array}$ & $\begin{array}{l}\text { Procedures } \\
\text { Open anastomosis (transplant) } \\
\text { Patient Care } \\
\text { Complication prevention and management } \\
\text { Complex ventilator issues } \\
\text { Complicated infections (esophageal leak, empyema, bronchial fistula) } \\
\text { Problem-based issues (hemoptysis, esophageal perforation) } \\
\text { Preop Issues } \\
\text { Operative decisions } \\
\text { Judgment } \\
\text { Salvage surgery (lung or esophageal cancer, GERD) } \\
\text { Intraop Issues } \\
\text { Anatomic lung resection } \\
\text { Thymectomy } \\
\text { Esophagectomy } \\
\text { Antireflux (open, laparoscopic, abdominal, thoracic) } \\
\text { Decortication } \\
\text { Reoperations/complex operations } \\
\text { Postop Issues } \\
\text { Outpatient problems } \\
\text { Long-term care }\end{array}$ \\
\hline Congenital cardiac & $\begin{array}{l}\text { Identification of curriculum and metrics } \\
\text { Finding centers of excellence } \\
\text { Team building } \\
\text { Anatomic models of congenital defects } \\
\text { Preop and postop care } \\
\text { Development of data repository }\end{array}$ & $\begin{array}{l}\text { Procedure simulation on models } \\
\text { Addition of physiologic modifiers to models } \\
\text { Introduction of new procedures }\end{array}$ \\
\hline
\end{tabular}

$C P B$, Cardiopulmonary bypass; intraop, intraoperative; $C A B G$, coronary artery bypass grafting; preop, preoperative; $M V$, mitral valve; ITA, internal thoracic artery; ICU, intensive care unit; $I C D$, implantable cardioverter defibrillator; $L V A D$, left ventricular assist device; $E G D$, esophagogastroduodenoscopy; $E B U S$, endobronchial ultrasonography; VATS, video-assisted thoracoscopic surgery; $O R$, operating room; GERD, gastroesophageal reflux disease; postop, postoperative. 
more common, it may become valuable to use simulation technology to teach the classic open procedures to residents. An example of such instruction involves laparoscopic cholecystectomy. It is rare today for surgical residents to experience open cholecystectomy except in the most complex and difficult cases, and the art of the classic operation may be lost to future generations of surgeons without simulation.

\section{Which team challenges and competencies most need to be simulated in the OR environment and in the critical care environment?}

The aviation industry learned early in its experience that the first step in simulating an environment was a thorough understanding of that environment. It is crucial to ensure that simulators are simulating reality, not just a perception of reality. The first step in simulating the OR environment for team training is therefore careful construction of the OR environment, its interactions, and its group dynamics. Once the environment has been defined, it will be possible to construct simulation environments that place each team member in defined roles and to develop multiple scenarios that the team can successfully complete. These scenarios should include both catastrophes and uncomplicated procedures. The goal is to empower each member to participate fully and to learn to avoid miscommunication at crucial times.

Participants agreed that the most dangerous time for errors to occur during the patient's hospitalization is the transport and handoff to the intensive care unit (ICU). Simulations of this activity should emphasize the importance of complete exchange of information about intraoperative events and patient-specific anatomy and physiology. The patient's baseline physiology and any changes consequent to the surgery should be understood by the critical care team. Problems encountered in the OR with drug sensitivity, bleeding, or tissue quality may be key components during the recovery period. Clear and precise communication is required between OR and ICU nurses, between anesthesia and critical care staff members, between surgeons and anesthesiologists, and among all combinations of these professionals.

Another component of critical care that is in dire need of simulation is crisis management. Recognition of equipment failure and appropriate management require regular training, as do emergency procedures. A crisis situation does not provide the best opportunity for learning to manage these challenges. Simulation of reentrant sternotomy or pulmonary resections complicated by acute hemorrhage could familiarize OR personnel with procedures and instrumentation before the need arises. Arrhythmias and their treatment could be simulated with currently available technology.

Recurring training with these models should be fundamental to the plan. Such training will require change in the current paradigm of practice to place value on participation in the simulation program. It will be necessary for teams to take time to run through these simulations, and it will be necessary for institutions and certifying boards to make this time available and ultimately required.

\section{What key simulation activities should happen, and in which locations?}

The distribution of simulation technology should be driven by a combination of the commonality of the procedure and the cost and complexity of the simulation. Table 2 summarizes this concept.

Fundamental skills universally required of all cardiothoracic surgeons, including common technical procedures, basic surgical techniques, and team-building exercises, should be available in local facilities. Local availability will allow broad distribution of low-fidelity, low-technology approaches that can keep costs at a reasonable level for all hospitals. Such an approach would permit successful implementation of mandatory requirements for resident education and advancement, hospital review, and approval of new privileges. Common surgical techniques such as suturing, placing chest tubes and central venous catheters, and performing vascular anastomoses, can be simulated with low-fidelity, low-cost models. Ex vivo bovine hearts can be used for practicing valvular procedures. Computer-based simulations can focus on surgically relevant anatomic relations, preoperative planning, and procedural steps for initiating and withdrawing CPB. Credentialing and advancement would be based on standardized levels of competence for each essential skill.

Two important foci for simulation at the local level are team building and crisis management. These areas are more effectively addressed at the point of action than by moving the team out of its environment to identify solutions that may not be locally relevant. Simple low-fidelity exercises highlighting team interaction and crisis management in the ICU, OR, catheterization laboratory, and ward can promote patient care and safety. Such exercises can also identify areas of stress management and team member behavior that can be modified for more efficient patient care and team dynamics. Computer-based or role-playing simulations of physician behaviors and interactions with patients and their family members can teach effective communication skills in difficult situations such as death or complications, unfavorable test results, or end-of-life discussions.

Table 2. Proposed distribution of simulation activities in various centers based on a combination of cost, complexity, and need

\begin{tabular}{lll}
\hline \multicolumn{1}{c}{ Skills } & \multicolumn{1}{c}{$\begin{array}{c}\text { Low-fidelity, } \\
\text { low cost }\end{array}$} & \multicolumn{1}{c}{$\begin{array}{c}\text { High-fidelity, } \\
\text { high cost }\end{array}$} \\
\hline Universal & Local centers & Mobile centers \\
Common & Regional centers & Regional centers \\
Uncommon or new & $\begin{array}{l}\text { Regional or mobile } \\
\text { centers }\end{array}$ & National center \\
\hline
\end{tabular}


This approach focuses on establishing competence in fundamental surgical skills and procedures that can be attained only by frequent repetition and ready availability. Competence would be required before advancement to more complicated activities at regional, mobile, or national levels, thus establishing ready criteria for competency-based advancement and national curriculum development while minimizing repetition and cost. The second benefit resides in the development of effective teams at the site of action and, rather than generalized solutions, offers personalized insight for local team building, identifies care issues specific to the local hospital environment, and can provide tool for OR debriefing, discussions between physicians and medical personnel in a nonconfrontational and nonhierarchical structure similar to that used by the airline industry.

Regional simulation activities should focus on teaching more complex skills or introducing new technologies that require more complex, higher-fidelity simulation. Regional simulation can also be used when there are too few users at the local level to justify the level of simulation required. Regional simulations could also serve as centers for maintenance of certification. Specific complex technology skills that the VSC recommended for regional implementation include surgical skills related to congenital heart surgery, VATS lobectomies and esophagectomies, implantation of ventricular assist devices, heart failure therapies, CPB scenarios, and endovascular techniques. In addition, training of simulation educators and research into simulation technology and curriculum would occur at these regional centers.

Similarly, mobile centers could focus on the delivery of higher-fidelity, higher-cost simulation of more complex technical or patient care issues. These facilities would be particularly suited to those situations for which local delivery remains important but cost and complexity preclude universal distribution.

Simulation at the national level would be limited to only a few high-fidelity centers at which developments in very complex specializations, skills, or innovations are required or where national certification could be maintained. These centers would focus on high-cost initiatives such as robotic surgery, uncommon or rare procedures, and new innovative technologies for which national validation studies and initial certification could be performed.

\section{Whose support and what resources are necessary to begin implementation of simulation in thoracic surgery, and how do we access them?}

Participants at the VSC focused on the key actions required for implementing simulation in three target areas:

1. Certification or maintenance of certification requires establishing specific competencies and a testable curriculum universal to all of cardiothoracic surgery, thereby establishing a quantifiable "industry standard." Competencies include fundamental surgical technologies and crucial procedures, such as anastomotic techniques, anatomic relationships, and CPB management. These competencies need to be constructed within a platform that minimizes the learning curve, given the limited time available for resident and surgeon education, and must be suitable for validation so that credentialing is possible.

2. Team training must focus on team building and group dynamics, behavior issues, identification of resource or system limitations, and error analysis. Simulation exercises will be focused on crucial areas in which competence is desired both for the individual surgeon and for the team as a whole, thus improving patient safety. Simulations will also serve to identify system or resource limitations and communication issues within a team. Second, each program or hospital will need to establish an internal system for deconstructing cases and simulating critical events with the goal of identifying a means of preventing errors or avoiding crises, establishing nonhierarchic pathways for conflict resolution among team members, increasing system adaptability and resilience for correcting system shortcomings and overcoming resource limitations, and defining team member priorities and roles, crisis guidelines, and algorithms. Implementation of even low-fidelity team simulations will require the development of trained facilitators with expertise in debriefing and case deconstruction. Retired faculty members may be a particularly valuable resource for serving as these experts.

3. Emerging new technologies are the costliest of the simulation activities because of the general requirement for higher-fidelity simulation, a high demand for keeping surgeons current in a competitive environment, and the rapid evolution of complex technologies. The level of simulation required for these activities is currently at or beyond the current state-of-the-art simulation, and future development will be necessary. It will be vitally important early on to identify key technologies that are perceived as "future standards of care" and to establish consensus guidelines, domain-specific training requirements, and competency levels. Gradual implementation of the truly key technologies will allow cost-effective curriculum development and certification for the standardized dissemination of new technologies within cardiothoracic surgery and provide a basis for possible recertification.

The support for this program was an essential theme of this discussion at the VSC, because patient safety is the basic foundation that both drives and justifies these efforts. The term support in this sense was defined either as financial, providing direct funding for development, or as legislative or mandatory, providing the requirement to proceed. With that premise, participants identified important sources of support that have not been traditionally considered.

Health care payers value improved safety and better outcomes. Ultimately, any effort that improves safety reduces 
adverse outcomes and costs. Thus the payer industry should be a source of financial and political support for the development of simulation. Members of the Leapfrog Group are already committed to actions that can improve patient safety and are driving a mandate to raise awareness and accountability for patient safety.

The medical device industry is already engaged in developing simulation. The incentives are multiple. The medical device industry is under FDA mandate to ensure proper use of devices, and there is marked aversion to litigation arising from incorrect use. A more effective partnership between the medical device industry and surgeons engaged in our professional organizations will lead to higher-fidelity simulation opportunities. These simulation systems can enhance the development of the next generation of devices. Ultimately, industry can expect to recoup some of the cost of development as hospitals and providers are required to use simulation under the auspices of improving patient safety.

Private and corporate investors are also a potential source of funding support. Although thoracic surgeons compose a small fraction of all health care providers, cardiovascular disease is a rapidly growing segment of the health care market, and thoracic oncology is an increasing clinical problem. Our professional organizations should partner with simulation developers to define specific needs and markets for simulation in thoracic surgery, to assess simulations that may be generalized to the broader health care industry, and to seek investor participation.

Software developers and publishers will have the potential to distribute software and to regularly update computer-based simulations. This industry may provide some funding support for the development of image-based simulations.

The academic medical center is a potential source of both financial and mandate support. There is a growing emphasis on "interprofessional" training centers that incorporate medical, dental, nursing, and pharmacy schools. Partnerships between academic schools and hospitals can build simulation platforms applicable to local environments, with the goal of reducing errors and thereby reducing costs and litigation risk.

Participants at VSC expressed the concern that the usual federal government funding sources, including the National Institutes of Health and Congress, do not provide fertile ground for new funding initiatives. Recent history reflects government acceptance of the need for improved patient safety, but these mandates have been largely unfunded. Less commonly considered federal resources might be better positioned to support medical simulation development. The Department of Defense (DOD) has some experience with simulation development and also has a history of funding health care initiatives that can improve battlefield care and survival. Simulation has great potential for preparing surgeons for the multitrauma care currently required for war injuries, which differs substantially from civilian trauma care. The need to place well-prepared surgeons in the field may be a powerful incentive for DOD support for the development of simulation. Similarly, the National Science Foundation traditionally supports engineering research. Although this organization has not previously provided substantial funding to the health care industry, the development of simulators will clearly be a feat of engineering. Finally, philanthropy can provide seed money to fund new, creative endeavors if we demonstrate the value of simulation in improving patient welfare.

The incentive to go forward with simulation development will require a mandate and coordination of educational activities. Within our profession, this mandate must come from the organizations responsible for defining educational goals and enforcing the requirements, such as the ABTS, the Residency Review Committee (RRC), and the TSDA, to set the standards for competence and professionalism.

In summary, supporting the development of simulation as an educational tool will require both a mandate and funding. Local institutions should begin these efforts without delay to use low-fidelity training programs. Continuing efforts can gradually build regional, national, and global programs.

\section{VSC: Outcomes and Next Steps}

At the conclusion of the conference, the representatives of each constituent organization met to discuss the actions their organizations would take to move forward. The following actions were defined.

\section{Prepare a Report From the VSC: TSFRE}

The conference organizing committee agreed to prepare a report from the VSC, which is represented by the current document. An abbreviated report was completed before the AATS meeting in May 2007 and was provided to each of the constituent organizations represented at the VSC. A draft of a complete manuscript was provided, with a goal of submitting the final report for simultaneous publication in Annals of Thoracic Surgery, Journal of Thoracic and Cardiovascular Surgery, European Journal of Thoracic Surgery, and CTSnet. The following representatives agreed to review the manuscript before final submission: JCTSE, Bill Baumgartner, MD; STS, Randolph Chitwood, MD; TSDA: Jay Zwischenberger, MD; AATS, Eugene Grossi, MD; ABTS, Richard Shemin, MD; EACTS, Lucio Parenzan, MD; and ACS, Ajit Sachdeva, MD.

\section{Create a Joint Task Force: TSFRE, JCTSE, AATS, STS, TSDA}

The directive given this task force was to oversee the progress in simulation in thoracic surgical education. The following activities would fall within the purview of the task force:

- Create an inventory or registry of cardiothoracic simulation.

- Technology readily available

- Technology under development

- Existing centers 
- Determine timeline, venue, cost, and format.

- Identify targets for high-fidelity simulation.

- Identify industry, government, and other partners.

- Foster research and education in this field.

- Facilitate development of funding.

Define Use of Simulation for Education, Certification, and Maintenance of Certification: ABTS, TSDA, TSRA

Representatives of these groups agreed that developing a directive or mandate to deploy simulation as a tool for thoracic surgical education fell within their purview. Specifically, the following activities are envisioned:

- Formulate simulator education as part of the thoracic surgical curriculum: TSDA.

- Prioritize procedures to be simulated: TSDA, TSRA.

- Define team training activities: TSDA, TSRA.

- Consider use of simulation for certification and maintenance of certification and define the necessary steps: ABTS.

\section{Actions Planned by Participant Organizations \\ Outside Thoracic Surgery: ACS}

During the conference, it became evident that the ACS has been pursuing simulation in surgical education for some time. It will be valuable to expand the College's informational efforts regarding the ACS Accredited Education
Institutes to ensure the best use of resources and collaboration across disciplines. ACS representatives agreed to expand information efforts regarding simulation activities that are widely applicable across disciplines, including communication, teamwork, team training, and professionalism. These collaborative efforts can be undertaken through the joint task force described previously to share resources and avoid duplication. This group will also investigate the possibility of establishing a mechanism whereby thoracic surgeons interested in simulation can convene with the leaders of the ACS-Accredited Institutes to discuss collaborative activities.

\section{Need for Specific Technology Training: Industry Representatives}

Industry is positioned to best determine generic need for training and the need for device-specific training as each industry partner develops technology. Collaborative independent programs funded by industry will be helpful and necessary, particularly in device development and proficiency.

TSFRE and the sponsoring surgical organizations acknowledge the outstanding assistance and support for the VSC by the Center for Medical Simulation at Harvard Medical School and Partners Healthcare. In particular, we thank Daniel Raemer, $\mathrm{PhD}$, who organizes the efforts at the Center for Medical Simulation and was enormously helpful in planning and executing this conference. 